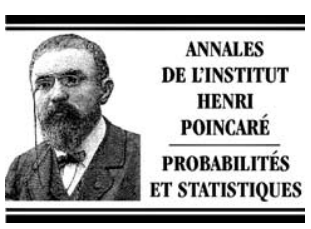

www.elsevier.com/locate/anihpb

\title{
Moments of last exit times for Lévy processes ${ }^{\text {th }}$
}

\author{
Ken-iti Sato ${ }^{\mathrm{a}, *}$, Toshiro Watanabe ${ }^{\mathrm{b}}$ \\ a Hachiman-yama 1101-5-103, Tenpaku-ku, Nagoya, 468-0074 Japan \\ ${ }^{\mathrm{b}}$ Center for Mathematical Sciences, the University of Aizu, Aizu-Wakamatsu, Fukushima, 965-8580 Japan \\ Received 23 January 2002; accepted 8 April 2003
}

\begin{abstract}
Let $L_{B_{a}}$ be the last exit time from the ball $B_{a}=\{|x|<a\}$ for a transient Lévy process $\left\{X_{t}\right\}$ on $\mathbb{R}^{d}$. It is proved that, for each $\eta \geqslant 0$, either $E\left[L_{B_{a}}{ }^{\eta}\right]<\infty$ for all $a>0$ or $E\left[L_{B_{a}}{ }^{\eta}\right]=\infty$ for all $a>0$. Let $\mathfrak{T}$ be the set of $\eta \geqslant 0$ having the former property. The size of $\mathfrak{T}$ gives an order of transience of $\left\{X_{t}\right\}$. A criterion for $\eta \in \mathfrak{T}$ is given in terms of the logarithm of the characteristic function of $X_{1}$. The set $\mathfrak{T}$ is determined when $d=1$ and $E\left[\left|X_{t}\right|\right]<\infty$. Examples and related results are given. (C) 2003 Elsevier SAS. All rights reserved.

Résumé

Soit $L_{B_{a}}$ le dernier temps de passage dans la boule $B_{a}=\{|x|<a\}$ pour un processus de Lévy transitoire $\left\{X_{t}\right\}$ à valeurs dans $\mathbb{R}^{d}$. On montre que pour tout $\eta \geqslant 0$, soit $E\left[L_{B_{a}}{ }^{\eta}\right]<\infty$ pour tout $a>0$, soit $E\left[L_{B_{a}}{ }^{\eta}\right]=\infty$ pour tout $a>0$. On considère l'ensemble $\mathfrak{T}$ des réels $\eta \geqslant 0$ qui vérifient la premiére propriété. La taille de $\mathfrak{T}$ permet de quantifier le caractère transitoire de $\left\{X_{t}\right\}$. Un critère pour que $\eta$ appartienne à $\mathfrak{T}$ est donné en termes du logarithme de la fonction caractéristique de $X_{1}$. L'ensemble $\mathfrak{T}$ est déterminé dans le cas où $d=1$ et $E\left[\left|X_{t}\right|\right]<\infty$. Des exemples et des résultats liés à celui-ci sont aussi donnés.
\end{abstract}

(c) 2003 Elsevier SAS. All rights reserved.

MSC: 60G51; 60G52

Keywords: Lévy process; Last exit time; Moment; Characteristic function

Mots-clés : Processus de Lévy ; Dernier temps de passage ; Moment ; Fonction caractéristique

\section{Introduction}

Let $\left\{X_{t}: t \geqslant 0\right\}$ be a Lévy process on $\mathbb{R}^{d}$, that is, a stochastically continuous process with stationary independent increments starting at the origin with cadlag sample functions a.s. For any Borel set $B$ let $L_{B}=\sup \left\{t \geqslant 0: X_{t} \in B\right\}$,

\footnotetext{
Research partly supported by the Grant-in-Aid for Scientific Research, No. 12640113, the Ministry of Education and Science, Japan.

* Corresponding author.

E-mail addresses: ken-iti.sato@nifty.ne.jp (K. Sato), t-watanb@u-aizu.ac.jp (T. Watanabe).
} 
the last exit time from $B$. It is easy to see the measurability of $L_{B}$ for open sets $B$. Let $B_{a}=\left\{x \in \mathbb{R}^{d}:|x|<a\right\}$. It is well known that either

$$
L_{B_{a}}=\infty \quad \text { a.s. for all } a>0
$$

or

$$
L_{B_{a}}<\infty \quad \text { a.s. for all } a>0 .
$$

The properties (1.1) and (1.2) are respectively called recurrence and transience. In this paper we study existence of moments of $L_{B_{a}}$ for a transient Lévy process $\left\{X_{t}\right\}$ on $\mathbb{R}^{d}$. The following are three main results.

1. For any $\eta>0$ we will show that either

$$
E\left[L_{B_{a}}{ }^{\eta}\right]<\infty \text { for all } a>0
$$

or

$$
E\left[L_{B_{a}}{ }^{\eta}\right]=\infty \text { for all } a>0 .
$$

In the proof we will use the fact that the support of $\left\{X_{t}\right\}$ is symmetric unless it is one-sided. Since (1.2) is assumed, we consider that (1.3) holds for $\eta=0$. We denote by $\mathfrak{T}$ the set of $\eta \geqslant 0$ such that (1.3) holds. The set $\mathfrak{T}$ is an interval with left end 0 or a singleton $\{0\}$. The size of $\mathfrak{T}$ measures an order of transience.

2. Let $\psi(z), z \in \mathbb{R}^{d}$, be the logarithm of the characteristic function of $X_{1}$, that is, the unique continuous function satisfying $\psi(0)=0$ and

$$
E\left[\mathrm{e}^{\mathrm{i}\left\langle z, X_{t}\right\rangle}\right]=\mathrm{e}^{t \psi(z)}
$$

In Bertoin [1] $-\psi(z)$ is called the characteristic exponent of $\left\{X_{t}\right\}$. We will give a criterion whether $\eta \in \mathfrak{T}$, using the function $\psi(z)$. This is a generalization of the Chung-Fuchs type criterion of recurrence and transience.

3. We will determine the set $\mathfrak{T}$ of a transient one-dimensional Lévy process $\left\{X_{t}\right\}$ with finite mean. It follows from transience that the mean is non-zero. The description of $\mathfrak{T}$ will be given in terms of finiteness or infiniteness of one-sided moments of $X_{t}$ or, equivalently, of its Lévy measure outside a neighborhood of the origin.

In a separate paper we will discuss the sets $\mathfrak{T}$ of transient stable and transient semi-stable processes on $\mathbb{R}^{d}$, using the results of this paper.

Last exit times for stable processes and Markov chains were discussed by Takeuchi, Yamada and Watanabe [23], Port [11-13], and Takeuchi [22] in 1960s in connection with probabilistic treatment of equilibrium measures. Then, for general transient Lévy processes they were discussed by Port and Stone [14]. Takeuchi [22] determined finiteness and infiniteness of the moments $E\left[L_{B_{a}}{ }^{\eta}\right]$ for rotation invariant $\alpha$-stable processes on $\mathbb{R}^{d}$. Following this, Hawkes [6] gave a criterion of finiteness and infiniteness of the moments in terms of the function $\psi(z)$ for symmetric one-dimensional Lévy processes. The symmetry assumption greatly simplifies the study. The criterion in the nonsymmetric case has not been treated so far.

The conditions (1.3) and (1.4) are respectively equivalent to the following properties (1.6) and (1.7):

$$
\begin{aligned}
& \int_{0}^{\infty} t^{\eta} P\left[X_{t} \in B_{a}\right] d t<\infty \quad \text { for all } a>0, \\
& \int_{0}^{\infty} t^{\eta} P\left[X_{t} \in B_{a}\right] d t=\infty \quad \text { for all } a>0 .
\end{aligned}
$$

A transient Lévy process is called strongly transient if (1.6) holds for $\eta=1$. Otherwise it is called weakly transient. Port [12] introduced this terminology for Markov chains and studied the influence of this distinction on limit theorems. Let $T_{B}$ be the hitting time of a compact set $B$. The observations on the first two terms in the asymptotic expansion for large $t$ of $\int_{\mathbb{R}^{d}} P^{x}\left[T_{B} \leqslant t\right] d x$ by Spitzer [21] for Brownian motions on $\mathbb{R}^{d}$ and by Getoor [5] for 
strictly stable processes are generalized by Port and Stone [14] to transient Lévy processes and recognized as the distinction between weak and strong transience. This is relevant also to limit theorems of ranges of random walks, as was shown by Jain and Pruitt [7]. Sato [16] gave a criterion of weak and strong transience analogous to the Chung-Fuchs type criterion of recurrence and transience and showed the non-existence of an analogue to Spitzer's criterion extended to Lévy processes by Port and Stone [14], Theorem 16.2. A paper [3] of Dawson, Gorostiza, and Wakolbinger uses a notion similar to the set $\mathfrak{T}$ and shows its importance in the analysis of branching systems.

This paper is organized in the following way. Section 2 proves result 1 . Criteria for $\eta \in \mathfrak{T}$ in terms of the function $\psi(z)$ (result 2) are given in Section 3. Some additional results and symmetric examples are provided in Section 4. Result 3 is given in Section 5. For a one-dimensional random walk $\left\{S_{n}: n=0,1, \ldots\right\}$ satisfying $S_{n} \rightarrow \infty$ a.s. as $n \rightarrow \infty$, a deep analysis of the moments of the last exit time $L_{(-\infty, a]}$ for $a \geqslant 0$ was made by Janson [8] and Kesten and Maller [9]. We rely on their works in Section 5. We give an example of a one-dimensional Lévy process $\left\{X_{t}\right\}$ satisfying $X_{t} \rightarrow \infty$ a.s. as $t \rightarrow \infty$ for which finiteness of $E\left[L_{B_{a}}{ }^{\eta}\right]$ is not equivalent to finiteness of $E\left[L_{(-\infty, a]}{ }^{\eta}\right]$.

\section{Dichotomy for moments of last exit times}

In this section we will prove result 1 stated in the previous section. First it will be proved in the case where the process has either symmetric or one-sided support. Then we will prove that the support of any Lévy process is either symmetric or one-sided. This result on the support is new to the best of our knowledge.

Let $\left\{X_{t}: t \geqslant 0\right\}$ be a Lévy process on $\mathbb{R}^{d}$. We consider it as the coordinate $X_{t}(\omega)=\omega(t)$ of $\omega$ in the space D of cadlag mappings from $[0, \infty)$ into $\mathbb{R}^{d}$. As usual $[1,2,18]\left\{X_{t}\right\}$ induces a unique Hunt process defined on $\left(\mathbf{D}, \mathcal{F}, \mathcal{F}_{t}, P^{x}: t \geqslant 0, x \in \mathbb{R}^{d}\right)$. The original process is identical in law with $\left\{X_{t}(\omega)\right\}$ under $P^{0}$. The process $\left\{X_{t}(\omega)\right\}$ under $P^{x}$ is identical in law with $\left\{x+X_{t}(\omega)\right\}$ under $P^{0}$. Let $\mu=\mathcal{L}\left(X_{1}\right)$, the distribution of $X_{1}$. Further let $\mu^{t}=\mathcal{L}\left(X_{t}\right), P_{t}(x, B)=P^{x}\left[X_{t} \in B\right]=\mu^{t}(B-x), U(B)=\int_{0}^{\infty} \mu^{t}(B) d t$, and $U(x, B)=U(B-x)$ for Borel sets $B$. For a function $f$ we use $\left(P_{t} f\right)(x)=\int P_{t}(x, d y) f(y)$ whenever the integral is defined. For $B$ in a class of sets including all $F_{\sigma}$-sets, the hitting time $T_{B}=\inf \left\{t>0: X_{t} \in B\right\}$ is an $\left\{\mathcal{F}_{t}\right\}$-stopping time and the last exit time $L_{B}$ is $\mathcal{F}$-measurable. Note that $\left\{L_{B}>t\right\}=\left\{T_{B} \circ \theta_{t}<\infty\right\}$, where $\theta_{t}$ is the shift of paths defined as $\left(\theta_{t} \omega\right)(s)=\omega(t+s)$. Let $K_{a}(x)=\left\{y \in \mathbb{R}^{d}:|y-x| \leqslant a\right\}$, the closed ball centered at $x$ with radius $a$, and let $K_{a}=K_{a}(0)$. The support $\Sigma$ is defined to be the smallest closed set satisfying $P^{0}\left[X_{t} \in \Sigma\right.$ for all $\left.t \geqslant 0\right]=1$ (see [18], Definition 24.13). A Lévy process on $\mathbb{R}^{d}$ is said to be one-sided if there is a vector $a \neq 0$ such that $\Sigma \subset\left\{x \in \mathbb{R}^{d}:\langle x, a\rangle \geqslant 0\right\}$. A Lévy process on $\mathbb{R}^{d}$ is said to be degenerate, if there are a proper linear subspace $V$ of $\mathbb{R}^{d}$ and a vector $a \in \mathbb{R}^{d}$ such that $P^{0}\left[X_{t} \in t a+V\right.$ for all $\left.t \geqslant 0\right]=1$; otherwise it is said to be nondegenerate (see [18], Definition 24.18). It is called genuinely $d$-dimensional if no proper linear subspace of $\mathbb{R}^{d}$ contains $\Sigma$.

In the following five lemmas we consider a transient Lévy process.

Lemma 2.1. Let $K$ and $K^{\prime}$ be compact sets such that the interior of $K^{\prime}$ contains $K$. Then there are finite positive constants $c_{1}$, c 2 such that, for all $x \in \mathbb{R}^{d}$,

$$
c_{1} P^{x}\left[T_{K}<\infty\right] \leqslant U\left(x, K^{\prime}\right) \leqslant c_{2} P^{x}\left[T_{K^{\prime}}<\infty\right] .
$$

This relation was suggested by T. Shiga and proved by Yamamuro [24], Lemma 2.2 and Remark 2.3.

Lemma 2.2. Let $K$ and $K^{\prime}$ be as in Lemma 2.1. Then there are finite positive constants $c_{3}, c_{4}$ such that, for all $\eta>0$ and $x \in \mathbb{R}^{d}$,

$$
c_{3} \int_{0}^{\infty} t^{\eta} P_{t}(x, K) d t \leqslant E^{x}\left[L_{K}{ }^{\eta}\right] \leqslant c_{4} \int_{0}^{\infty} t^{\eta} P_{t}\left(x, K^{\prime}\right) d t .
$$


Proof. Notice that

$$
E^{x}\left[L_{K}^{\eta}\right]=\int_{0}^{\infty} P^{x}\left[L_{K}>t\right] \eta t^{\eta-1} d t=\int_{0}^{\infty} E^{x}\left[P^{X_{t}}\left[T_{K}<\infty\right]\right] \eta t^{\eta-1} d t .
$$

Hence, by Lemma 2.1, there are $c_{3}$ and $c_{4}$ such that

$$
c_{3} \int_{0}^{\infty} E^{x}\left[U\left(X_{t}, K\right)\right] \eta t^{\eta-1} d t \leqslant E^{x}\left[L_{K}^{\eta}\right] \leqslant c_{4} \int_{0}^{\infty} E^{x}\left[U\left(X_{t}, K^{\prime}\right)\right] \eta t^{\eta-1} d t .
$$

This is (2.2), since

$$
\int_{0}^{\infty} E^{x}\left[U\left(X_{t}, K\right)\right] \eta t^{\eta-1} d t=\int_{0}^{\infty} E^{x}\left[\int_{t}^{\infty} 1_{K}\left(X_{s}\right) d s\right] \eta t^{\eta-1} d t=\int_{0}^{\infty} s^{\eta} P_{s}(x, K) d s
$$

and similarly for $K^{\prime}$.

In the case of $\eta=1$, the next lemma is by Port [11,12] and Yamamuro [24].

Lemma 2.3. Let $\eta>0$. Then (1.3) is equivalent to (1.6) and (1.4) is equivalent to (1.7).

Proof. The assertion is a direct consequence of Lemma 2.2. Note that the expectation and the two integrals in (2.2) are not assumed to be finite.

Lemma 2.4. Let $\eta>0$. If the support $\Sigma$ is symmetric (that is, $\Sigma=-\Sigma$ ), then $\eta$ satisfies either (1.3) or (1.4).

Proof. Suppose that (1.4) does not hold. Then, by Lemma 2.3, there is $a>0$ such that $\int_{0}^{\infty} t^{\eta} P_{t}\left(0, K_{a}\right) d t<\infty$. We claim that, for every $x \in \mathbb{R}^{d}$,

$$
\int_{0}^{\infty} t^{\eta} P_{t}\left(0, K_{a / 2}(x)\right) d t<\infty
$$

Indeed, assume that the integral in (2.3) is positive. Then $P^{0}\left[T_{K_{a / 2}(x)}<\infty\right]>0$. Hence, by the symmetry of $\Sigma$, we have $P^{0}\left[T_{F}<\infty\right]>0$ for $F=K_{a / 2}(-x)$. Since

$$
\begin{aligned}
\int_{0}^{\infty} t^{\eta} P_{t}\left(0, K_{a}\right) d t & \geqslant E^{0}\left[\int_{0}^{\infty} t^{\eta} 1_{K_{a}}\left(X_{t} \circ \theta_{T_{F}}\right) d t ; T_{F}<\infty\right] \\
& =E^{0}\left[E^{X\left(T_{F}\right)}\left[\int_{0}^{\infty} t^{\eta} 1_{K_{a}}\left(X_{t}\right) d t\right] ; T_{F}<\infty\right]
\end{aligned}
$$

and, for any $y \in F$,

$$
E^{y}\left[\int_{0}^{\infty} t^{\eta} 1_{K_{a}}\left(X_{t}\right) d t\right]=E^{0}\left[\int_{0}^{\infty} t^{\eta} 1_{K_{a}}\left(y+X_{t}\right) d t\right] \geqslant E^{0}\left[\int_{0}^{\infty} t^{\eta} 1_{K_{a / 2}(x)}\left(X_{t}\right) d t\right],
$$


we obtain

$$
\int_{0}^{\infty} t^{\eta} P_{t}\left(0, K_{a}\right) d t \geqslant \int_{0}^{\infty} t^{\eta} P_{t}\left(0, K_{a / 2}(x)\right) d t P^{0}\left[T_{F}<\infty\right] .
$$

This shows (2.3). Now, for any $b>0, K_{b}$ is covered by a finite number of sets $K_{a / 2}(x), x \in K_{b}$. Hence $\int_{0}^{\infty} t^{\eta} P_{t}\left(0, K_{b}\right) d t<\infty$. That is, (1.3) holds by Lemma 2.3.

Lemma 2.5. Consider a one-sided transient Lévy process on $\mathbb{R}^{d}$. Then all $\eta>0$ satisfy (1.3).

Proof. We may and do assume that $\left\{X_{t}\right\}$ is genuinely $d$-dimensional. Assume that $d=1$. Then $\left\{X_{t}\right\}$ or $\left\{-X_{t}\right\}$ is a subordinator. Consider the former case (the latter case is similar). Then, for every $a>0$, we can find a constant $c>0$ such that

$$
P^{0}\left[X_{t} \leqslant a\right] \leqslant \mathrm{e}^{-c t} \text { for all large } t .
$$

Indeed, if $\left\{X_{t}\right\}$ is a deterministic motion, then (2.4) is obvious. If $\left\{X_{t}\right\}$ is not a deterministic motion, then $P^{0}\left[X_{1} \leqslant a\right]=\mathrm{e}^{-c^{\prime}}$ with some $c^{\prime}>0$ ([18], Theorem 24.3). Hence $P^{0}\left[X_{n} \leqslant a\right] \leqslant \mathrm{e}^{-c^{\prime} n}$ and, for large $t$, $P^{0}\left[X_{t} \leqslant a\right] \leqslant P^{0}\left[X_{n} \leqslant a\right] \leqslant \mathrm{e}^{-c^{\prime} n} \leqslant \mathrm{e}^{-c^{\prime}(t-1)} \leqslant \mathrm{e}^{-c t}$ with some $c>0$, where $n \leqslant t<n+1$. For any $\eta>0$, (1.6) follows from (2.4), hence we have (1.3).

Let $d \geqslant 2$. There is $x_{0} \neq 0$ such that $\left\{\left\langle x_{0}, X_{t}\right\rangle: t \geqslant 0\right\}$ is a non-zero subordinator. Given $a>0$, let $F=$ $\left\{y:\left\langle y, x_{0}\right\rangle \leqslant a\left|x_{0}\right|\right\}$. Then $K_{a} \subset F$ and hence $L_{K_{a}} \leqslant L_{F}$. Thus $\left\{X_{t}\right\}$ satisfies (1.3) by the result in the 1-dimensional case.

Given linearly independent vectors $x_{1}, \ldots, x_{d}$ in $\mathbb{R}^{d}$, we denote

$$
C\left(x_{1}, \ldots, x_{d}\right)=\left\{y=a_{1} x_{1}+\cdots+a_{d} x_{d}: a_{j}>0 \text { for } 1 \leqslant j \leqslant d\right\},
$$

the open convex cone generated by $x_{1}, \ldots, x_{d}$.

Lemma 2.6. Let $\left\{X_{t}\right\}$ be a Lévy process on $\mathbb{R}^{d}$ which is not one-sided.

(i) If $x_{1}, \ldots, x_{d}$ are linearly independent vectors in the support $\Sigma$, then, for every $y \in C\left(x_{1}, \ldots, x_{d}\right)$ and for every $\varepsilon>0$, there exist $w \in \Sigma$ and a positive integer $n$ such that $|n y-w|<\varepsilon$.

(ii) For every $y \in \mathbb{R}^{d} \backslash\{0\}$, we can find linearly independent vectors $x_{1}, \ldots, x_{d}$ in $\Sigma$ such that $y \in C\left(x_{1}, \ldots, x_{d}\right)$.

Proof. (i) First notice that $\Sigma$ is closed under addition ([18], Proposition 24.14). Let $x_{1}, \ldots, x_{d}$ be linearly independent vectors in $\Sigma$ and let $y=a_{1} x_{1}+\cdots+a_{d} x_{d}$ with $a_{j}>0$. If all of $a_{1}, \ldots, a_{d}$ are rationals, then there are positive integers $n_{0}, n_{1}, \ldots, n_{d}$ such that $n_{0} y=n_{1} x_{1}+\cdots+n_{d} x_{d}$, which gives the lemma, since $n_{1} x_{1}+\cdots+n_{d} x_{d}$ belongs to $\Sigma$. If at least one of $a_{1}, \ldots, a_{d}$ is irrational, then, for every $\varepsilon^{\prime}>0$, there are a positive integer $n$ and integers $n_{1}, \ldots, n_{d}$ such that $\left|n y-\left(n_{1} x_{1}+\cdots+n_{d} x_{d}\right)\right|<\varepsilon^{\prime}$ by the ergodicity of an irrational translation of the $d$-dimensional torus (see Petersen [10], p. 51). Choosing $\varepsilon^{\prime}$ sufficiently small, we see that $n_{1}, \ldots, n_{d}$ are positive and hence $n_{1} x_{1}+\cdots+n_{d} x_{d} \in \Sigma$.

(ii) We notice that, given $y \in \mathbb{R}^{d}$ and linearly independent vectors $z_{1}, \ldots, z_{d}$ in $\mathbb{R}^{d}$, there is $\varepsilon>0$ such that when $z_{1}^{\prime}, \ldots, z_{d}^{\prime}$ move in the $\varepsilon$-neighborhoods of $z_{1}, \ldots, z_{d}$, respectively, $z_{1}^{\prime}, \ldots, z_{d}^{\prime}$ are linearly independent and the coefficients $a_{1}^{\prime}, \ldots, a_{d}^{\prime}$ in the representation $y=a_{1}^{\prime} z_{1}^{\prime}+\cdots+a_{d}^{\prime} z_{d}^{\prime}$ are continuous functions of $z_{1}^{\prime}, \ldots, z_{d}^{\prime}$. Let $C_{0}$ be the union of $C\left(x_{1}, \ldots, x_{d}\right)$ with $x_{1}, \ldots, x_{d}$ running over all linearly independent systems of vectors in $\Sigma$. Then $C_{0}$ is an open set. Our assertion is that $C_{0}=\mathbb{R}^{d} \backslash\{0\}$. Let $z_{1}, \ldots, z_{d}$ be linearly independent vectors in $C_{0}$. We claim that

$$
C\left(z_{1}, \ldots, z_{d}\right) \subset C_{0} .
$$


Indeed, for any $\varepsilon>0$, we can find $c_{j}>0$ and $w_{j} \in \Sigma$ for $1 \leqslant j \leqslant d$ such that $\left|z_{j}-c_{j} w_{j}\right|<\varepsilon$, using the assertion (i). Given $y \in C\left(z_{1}, \ldots, z_{d}\right)$, we see that $w_{1}, \ldots, w_{d}$ are linearly independent and $y \in C\left(w_{1}, \ldots, w_{d}\right)$ if $\varepsilon$ is small. This shows that (2.5) is true. Next, we claim that $C_{0} \cup\{0\}$ is convex, that is, for any $z_{1}$ and $z_{2}$ in $C_{0} \cup\{0\}$,

$$
p z_{1}+(1-p) z_{2} \in C_{0} \cup\{0\} \text { for } 0 \leqslant p \leqslant 1 .
$$

If $z_{1}$ and $z_{2}$ are linearly dependent, then this is obvious. If $z_{1}$ and $z_{2}$ are linearly independent, then, choose $z_{3}, \ldots, z_{d}$ in $C_{0}$ such that $z_{1}, \ldots, z_{d}$ are linearly independent (this is possible because $C_{0}$ is open) and note that, letting $z_{j}^{\prime}=z_{j}-\varepsilon z_{3}-\cdots-\varepsilon z_{d}$ for $j=1,2$, the vectors $z_{1}^{\prime}, z_{2}^{\prime}, z_{3}, \ldots, z_{d}$ are linearly independent and in $C_{0}$ for small $\varepsilon>0$, and that

$$
p z_{1}+(1-p) z_{2}=p z_{1}^{\prime}+(1-p) z_{2}^{\prime}+\varepsilon z_{3}+\cdots+\varepsilon z_{d} \in C\left(z_{1}^{\prime}, z_{2}^{\prime}, z_{3}, \ldots, z_{d}\right) \quad \text { for } 0<p<1,
$$

implying (2.6) by using (2.5) for $z_{1}^{\prime}, z_{2}^{\prime}, z_{3}, \ldots, z_{d}$. It follows that $C_{0} \cup\{0\}$ is a convex cone. For any $x_{1} \in \Sigma$, we can find $x_{2}, \ldots, x_{d} \in \Sigma$ such that $x_{1}, \ldots, x_{d}$ are linearly independent (because $\left\{X_{t}\right\}$ is not one-sided), and hence $x_{1}$ belongs to the closure of $C\left(x_{1}, \ldots, x_{d}\right)$. Thus $\Sigma$ is contained in the closure of $C_{0} \cup\{0\}$. It follows that $C_{0} \cup\{0\}$ is not contained in any half space. Now we have only to recall the fact that any convex cone which is not contained in a half space coincides with the whole space $\mathbb{R}^{d}$ (Rockafellar [15], Corollary 11.7.3).

Theorem 2.7. If $\left\{X_{t}\right\}$ is a Lévy process on $\mathbb{R}^{d}$ which is not one-sided, then the support $\Sigma$ of $\left\{X_{t}\right\}$ is symmetric.

Proof. Let $\left\{X_{t}\right\}$ be not one-sided. Let $y \in \Sigma$ and $y \neq 0$. Applying Lemma 2.6(ii) to $-y$, we can find linearly independent vectors $x_{1}, \ldots, x_{d}$ in $\Sigma$ such that $-y \in C\left(x_{1}, \ldots, x_{d}\right)$. Now let us use Lemma 2.6(i). For any $\varepsilon>0$, there are $w \in \Sigma$ and a positive integer $n$ such that $|-n y-w|<\varepsilon$, that is, $|-y-((n-1) y+w)|<\varepsilon$. Since $\Sigma$ is closed under addition, $(n-1) y+w$ is in $\Sigma$. Since $\Sigma$ is a closed set and since $\varepsilon$ is arbitrary, we see that $-y \in \Sigma$. Hence $-\Sigma \subset \Sigma$, that is, $-\Sigma=\Sigma$.

In the proof we have, in fact, shown the following: if a subset $\Sigma$ of $\mathbb{R}^{d}$ is closed under convergence and addition, then either $\Sigma$ is contained in a half space $\{x:\langle x, a\rangle \geqslant 0\}, a \neq 0$, or $\Sigma$ is symmetric.

Theorem 2.8. Let $\left\{X_{t}\right\}$ be a transient Lévy process on $\mathbb{R}^{d}$. Then each $\eta>0$ satisfies either (1.3) or (1.4).

Proof. Combine Lemmas 2.4 and 2.5 with Theorem 2.7.

Since $1 \in \mathfrak{T}$ is equivalent to strong transience, we get the following.

Corollary 2.9. Any transient Lévy process on $\mathbb{R}^{d}$ is either weakly or strongly transient.

\section{Criteria in terms of the function $\psi(z)$}

Let $\left\{X_{t}\right\}$ be a transient Lévy process on $\mathbb{R}^{d}$. First we will give a useful sufficient condition for $\eta \in \mathfrak{T}$ using $|\psi(z)|$ and discuss its consequence (Theorem 3.2 and Corollary 3.3). If $\left\{X_{t}\right\}$ is symmetric (that is, $\left\{X_{t}\right\}$ and $\left\{-X_{t}\right\}$ are identical in law), then the function $\psi(z)$ is real, and it is easy to find a criterion whether $\eta \in \mathfrak{T}$, which will be formulated in Theorem 3.4. In a general nonsymmetric case, we give a criterion in Theorem 3.5 under the strong non-lattice condition.

We use two functions $f(x)$ and $g(z)$ given by 


$$
\begin{aligned}
& f(x)=f_{b}(x)=\prod_{j=1}^{d} \frac{\left(\sin b x_{j}\right)^{2}}{\left(b x_{j}\right)^{2}} \\
& g(z)=g_{b}(z)=\left(\frac{\pi}{b}\right)^{d} \prod_{j=1}^{d}\left(1-\frac{\left|z_{j}\right|}{2 b}\right) 1_{[-2 b, 2 b]}\left(z_{j}\right),
\end{aligned}
$$

where $x=\left(x_{j}\right)_{1 \leqslant j \leqslant d} \in \mathbb{R}^{d}, z=\left(z_{j}\right)_{1 \leqslant j \leqslant d} \in \mathbb{R}^{d}$, and $b>0$. We have

$$
(F f)(z)=g(z), \quad(F g)(x)=(2 \pi)^{d} f(-x)=(2 \pi)^{d} f(x),
$$

where $F$ denotes the the Fourier transform, $(F f)(z)=\int_{\mathbb{R}^{d}} \mathrm{e}^{\mathrm{i}\langle z, x\rangle} f(x) d x$.

Lemma 3.1. For any Lévy process on $\mathbb{R}^{d}$, we have, for $q>0$ and $\eta \geqslant 0$,

$$
\begin{aligned}
& \int_{0}^{\infty} \mathrm{e}^{-q t} t^{\eta}\left(P_{t} f\right)(0) d t=(2 \pi)^{-d} \Gamma(\eta+1) \int_{\mathbb{R}^{d}} g(z)(q-\psi(z))^{-\eta-1} d z, \\
& \int_{0}^{\infty} \mathrm{e}^{-q t} t^{\eta}\left(P_{t} g\right)(0) d t=\Gamma(\eta+1) \int_{\mathbb{R}^{d}} f(z)(q-\psi(z))^{-\eta-1} d z,
\end{aligned}
$$

where we understand $(q-\psi(z))^{-\eta-1}=|q-\psi(z)|^{-\eta-1} \mathrm{e}^{\mathrm{i}(-\eta-1) \arg (q-\psi(z))}$ with $\arg (q-\psi(z)) \in(-\pi / 2, \pi / 2)$.

Proof. Using (1.5), (3.3), and Fubini's theorem, we get

$$
\int_{0}^{\infty} \mathrm{e}^{-q t} t^{\eta}\left(P_{t} f\right)(0) d t=(2 \pi)^{-d} \int g(z) d z \int_{0}^{\infty} \mathrm{e}^{-t(q-\psi(z))} t^{\eta} d t .
$$

Notice that, for $w \in \mathbb{C}$ with $\operatorname{Re} w>0$, we have

$$
\int_{0}^{\infty} \mathrm{e}^{-t w} t^{\eta} d t=\Gamma(\eta+1) w^{-\eta-1}
$$

where $w^{-\eta-1}=|w|^{-\eta-1} \mathrm{e}^{\mathrm{i}(-\eta-1) \arg w}$ with $\arg w \in(-\pi / 2, \pi / 2)$. Now we get the first identity of the lemma, since $\operatorname{Re} \psi(z) \leqslant 0$. The second one is proved similarly.

Theorem 3.2. Let $\left\{X_{t}\right\}$ be a Lévy process on $\mathbb{R}^{d}$. Let $\eta \geqslant 0$. If

$$
\int_{|z|<\varepsilon}|\psi(z)|^{-\eta-1} d z<\infty
$$

for some $\varepsilon>0$, then $\left\{X_{t}\right\}$ is transient and $\eta \in \mathfrak{T}$.

Proof. We use the first identity in Lemma 3.1. Choose $0<b<\varepsilon /(2 \sqrt{d})$. Then $[-2 b, 2 b]^{d} \subset B_{\varepsilon}$. We have $|q-\psi|^{-\eta-1} \leqslant|\psi|^{-\eta-1}$, since $\operatorname{Re} \psi \leqslant 0$. Hence

$$
\underset{q \downarrow 0}{\limsup }\left|\int_{\mathbb{R}^{d}} g_{b}(z)(q-\psi(z))^{-\eta-1} d z\right| \leqslant \mathrm{const} \int_{B_{\varepsilon}}|\psi|^{-\eta-1} d z<\infty .
$$


It follows that $\int_{0}^{\infty} t^{\eta}\left(P_{t} f_{b}\right)(0) d t<\infty$. We have $f_{b}(x)>0$ if $\max _{1 \leqslant j \leqslant d}\left|x_{j}\right|<\pi / b$. Hence, for any $a>0$, there are $b>0$ and $c>0$ such that $f_{b} \geqslant c 1_{K_{a}}$. Thus (1.6) holds, which gives transience and $\eta \in \mathfrak{T}$.

Genuinely $d$-dimensional Lévy processes on $\mathbb{R}^{d}$ are transient if $d \geqslant 3$; they are strongly transient if $d \geqslant 5$ ([17], Theorem 2.17 and [18], Theorem 37.8). These facts are generalized in the following.

Corollary 3.3. If $\left\{X_{t}\right\}$ is genuinely $d$-dimensional and $d \geqslant 3$, then $[0, d / 2-1) \subset \mathfrak{T}$.

Proof. We use the generating triplet $(A, v, \gamma)$ in the Lévy-Khintchine representation of $\psi(z)$ ([18], Definition 8.2). Here $A$ is the Gaussian covariance matrix, $v$ is the Lévy measure, and $\gamma$ is a location parameter. Let $W$ be the smallest linear subspace that contains both $A\left(\mathbb{R}^{d}\right)$ and the support of $v$. Since $\left\{X_{t}\right\}$ is genuinely $d$-dimensional, there is no proper linear subspace that contains $W$ and $\gamma$. Assume that $W=\mathbb{R}^{d}$. Then $\left\{X_{t}\right\}$ is nondegenerate and there are $c>0$ and $\varepsilon>0$ such that

$$
|\hat{\mu}(z)| \leqslant 1-c|z|^{2} \quad \text { for }|z|<\varepsilon
$$

by [18], Proposition 24.19, and thus

$$
-\operatorname{Re} \psi(z) \geqslant c^{\prime}|z|^{2} \quad \text { for }|z|<\varepsilon
$$

with some $c^{\prime}>0$. Hence

$$
\int_{|z|<\varepsilon}|\psi|^{-\eta-1} d z \leqslant \text { const } \int_{|z|<\varepsilon}|z|^{-2(\eta+1)} d z=\mathrm{const} \int_{0}^{\varepsilon} r^{d-2 \eta-3} d r<\infty
$$

if $0 \leqslant \eta<d / 2-1$. Thus $[0, d / 2-1) \subset \mathfrak{T}$ by Theorem 3.2 .

On the other hand, assume that $W$ is $(d-1)$-dimensional. Then $\gamma \notin W$ and $\left\{X_{t}-t \gamma\right\}$ is a Lévy process on $W$. Let $\gamma=\gamma_{1}+\gamma_{2}$, where $\gamma_{1} \in W$ and $\gamma_{2} \in W^{\perp}$. Then $\gamma_{2} \neq 0$ and

$$
\left|X_{t}\right|^{2}=\left|X_{t}-t \gamma+t \gamma_{1}\right|^{2}+\left|t \gamma_{2}\right|^{2} \geqslant t^{2}\left|\gamma_{2}\right|^{2} \text {. }
$$

Hence $L_{K_{a}} \leqslant a /\left|\gamma_{2}\right|$ a.s., which shows that $\mathfrak{T}=[0, \infty)$.

Theorem 3.4. Consider a symmetric transient Lévy process on $\mathbb{R}^{d}$. Let $\eta \geqslant 0$ and $\varepsilon>0$. Then $\eta \in \mathfrak{T}$ if and only if

$$
\int_{|z|<\varepsilon}(-\psi(z))^{-\eta-1} d z<\infty \text {. }
$$

A result close to this theorem was given by Hawkes [6]. But his proof was more complicated. A simple example for this theorem is a rotation invariant stable process $\left\{X_{t}\right\}$ on $\mathbb{R}^{d}, d \geqslant 1$, with index $\alpha, 0<\alpha \leqslant 2$. Then $\psi(z)=-c|z|^{\alpha}$ with some $c>0$. It is transient if and only if $\alpha<d$. If $\alpha<d$, then $\mathfrak{T}=\left[0, \frac{d}{\alpha}-1\right)$. Brownian motion $(\alpha=2, d \geqslant 3)$ has the smallest set $\mathfrak{T}$.

Proof of Theorem 3.4. If $\eta=0$, then (3.8) is true (see [18], Corollary 37.6). Let $\eta>0$. If $\eta \in \mathfrak{T}$, then, by (1.6), $\int_{0}^{\infty} t^{\eta}\left(P_{t} g_{b}\right)(0) d t<\infty$ for all $b>0$, while the second identity of Lemma 3.1 with $q \downarrow 0$ shows that

$$
\int_{0}^{\infty} t^{\eta}\left(P_{t} g_{b}\right)(0) d t=\Gamma(\eta+1) \int_{\mathbb{R}^{d}} f_{b}(z)(-\psi(z))^{-\eta-1} d z
$$


which implies (3.8), as we have $\inf _{z \in B_{\varepsilon}} f_{b}(z)>0$ by taking $b$ small enough. On the other hand, if (3.8) holds, then $\int_{\mathbb{R}^{d}} g_{b}(z)(-\psi(z))^{-\eta-1} d z$ is finite when $b$ is taken small enough, and by Lemma 3.1

$$
\int_{0}^{\infty} t^{\eta}\left(P_{t} f_{b}\right)(0) d t=(2 \pi)^{-d} \Gamma(\eta+1) \int_{\mathbb{R}^{d}} g_{b}(z)(-\psi(z))^{-\eta-1} d z<\infty,
$$

which shows that $\int_{0}^{\infty} t^{\eta} P_{t}\left(0, B_{a}\right) d t<\infty$ for $a$ small enough, that is, $\eta \in \mathfrak{T}$ by Lemma 2.3 .

We introduce the following functions. Let $f_{1}(x)$ be the function in (3.1) with $b=1$. Then

$$
f_{1}(x)=\sum_{l=0}^{\infty} h_{l}(x)
$$

where

$$
h_{l}(x)=\sum_{k \in \mathbb{Z}_{+}^{d},\|k\|=l} c_{k} x_{1}^{2 k_{1}} \ldots x_{d}^{2 k_{d}}, \quad l=0,1, \ldots,
$$

with some $c_{k}$. Here $\|k\|=k_{1}+\cdots+k_{d}$ for $k=\left(k_{j}\right)_{1 \leqslant j \leqslant d} \in \mathbb{Z}_{+}^{d}$. Since

$$
f_{1}(x)=\prod_{j=1}^{d} \frac{1-\cos 2 x_{j}}{2 x_{j}^{2}}=\prod_{j=1}^{d}\left(1-\frac{2^{3} x_{j}^{2}}{4 !}+\frac{2^{5} x_{j}^{4}}{6 !}-\cdots\right),
$$

we see that

$$
h_{0}(x)=1, \quad h_{1}(x)=-\frac{1}{3}|x|^{2}, \quad h_{2}(x)=\frac{2^{5}}{6 !} \sum_{j=1}^{d} x_{j}^{4}+\frac{2^{7}}{(4 !)^{2}} \sum_{j_{1}<j_{2}} x_{j_{1}}^{2} x_{j_{2}}^{2},
$$

and so on. If $d=1$, then

$$
h_{l}(x)=(-1)^{l} \frac{2^{2 l+1}}{(2 l+2) !} x^{2 l}, \quad x \in \mathbb{R}, l=0,1, \ldots .
$$

A Lévy process $\left\{X_{t}\right\}$ on $\mathbb{R}^{d}$ is said to be strongly non-lattice if $\lim \sup _{|z| \rightarrow \infty}\left|\mathrm{e}^{\psi(z)}\right|<1$.

Theorem 3.5. Assume that $\left\{X_{t}\right\}$ is a strongly non-lattice, transient Lévy process on $\mathbb{R}^{d}$. Let $\eta>0$. Fix $\varepsilon>0$. Then $\eta \in \mathfrak{T}$ if and only if

$$
\underset{q \downarrow 0}{\limsup }\left|\int_{|z|<\varepsilon} h_{l}(z)(q-\psi(z))^{-\eta-1} d z\right|<\infty
$$

for $l=0,1, \ldots, N-1$, where $N$ is the positive integer satisfying $N-1<\eta \leqslant N$.

In (3.12) the function $(q-\psi(z))^{-\eta-1}$ is defined for $z \in \mathbb{R}^{d}$ as in Lemma 3.1.

We do not know whether Theorem 3.5 is true without the strong non-lattice assumption. Another question to which we do not have an answer is whether (3.12) for $l=0$ suffices for $\eta>0$ to belong to $\mathfrak{T}$.

We use the following lemma.

Lemma 3.6. There does not exist a finite system of infinite sequences of complex numbers $\left\{x_{l, n}: n=1,2, \ldots\right\}$, $l=1,2, \ldots, N$, having the following property: there is $\varepsilon>0$ such that, for all $b \in(0, \varepsilon), \lim _{n \rightarrow \infty} \sum_{l=1}^{N} b^{l} x_{l, n}=1$. 
Proof. If $N=1$, the assertion is obvious, because $\left\{x_{1, n}\right\}$ with the property above satisfies $\lim _{n \rightarrow \infty} x_{1, n}=1 / b$ for all $b \in(0, \varepsilon)$, which is absurd. Assume that $N \geqslant 2$ and that the assertion is true for $N-1$ in place of $N$. Suppose that $\left\{x_{l, n}\right\}, l=1,2, \ldots, N$, with the property above exist. If a subsequence $\left\{x_{1, n^{\prime}}\right\}$ of $\left\{x_{1, n}\right\}$ satisfies that $\left|x_{1, n^{\prime}}\right| \rightarrow \infty$, then $y_{l, n^{\prime}}=x_{l, n^{\prime}} / x_{1, n^{\prime}}$ satisfies $b+\sum_{l=2}^{N} b^{l} y_{l, n^{\prime}} \rightarrow 0$, that is, $-\sum_{l=1}^{N-1} b^{l} y_{l+1, n^{\prime}} \rightarrow 1$, which is impossible by the assumption. Hence $\left\{x_{1, n}\right\}$ is bounded. Next, if a subsequence $\left\{x_{2, n^{\prime}}\right\}$ of $\left\{x_{2, n}\right\}$ satisfies that $\left|x_{2, n^{\prime}}\right| \rightarrow \infty$, then, for $z_{l, n^{\prime}}=x_{l, n^{\prime}} / x_{2, n^{\prime}}$, we have $b^{2}+\sum_{l=3}^{N} b^{l} z_{l, n^{\prime}} \rightarrow 0$, which is again impossible. Hence $\left\{x_{2, n}\right\}$ is bounded. Repeating this, we see that $\left\{x_{1, n}\right\}, \ldots,\left\{x_{N, n}\right\}$ are all bounded. Now we can choose $0<n_{1}<n_{2}<\cdots$ such that, for all $l,\left\{x_{l, n_{k}}\right\}$ tends to some finite $c_{l}$. Then we have $\sum_{l=1}^{N} b^{l} c_{l}=1$ for $b \in(0, \varepsilon)$, which contradicts the fact that a polynomial of degree $N$ has at most $N$ real roots.

Proof of Theorem 3.5. Let us prove that $\eta$ satisfies (1.6) if and only if it satisfies (3.12) for $l=0,1, \ldots, N-1$. Assume that it satisfies (1.6). Using $g_{b}(z)$ in (3.2), this implies that

$$
\int_{0}^{\infty} t^{\eta}\left(P_{t} g_{b}\right)(0) d t<\infty \quad \text { for } b>0 \text {. }
$$

Hence, by Lemma 3.1,

$$
\underset{q \downarrow 0}{\limsup }\left|\int_{\mathbb{R}^{d}} f_{b}(z)(q-\psi(z))^{-\eta-1} d z\right|<\infty \quad \text { for } b>0 .
$$

We have

$$
f_{b}(z)=f_{1}(b z)=\sum_{l=0}^{N-1} b^{2 l} h_{l}(z)+b^{2 N}|z|^{2 N} H_{N}(b z),
$$

where $H_{N}(z)$ is a bounded function on $\mathbb{R}^{d}$. We denote $\psi_{1}(z)=\operatorname{Re} \psi(z)$ and $\psi_{2}(z)=\operatorname{Im} \psi(z)$. The strong nonlattice property means the existence of $c_{1}>0$ such that

$$
-\psi_{1}(z)>c_{1} \quad \text { for all large }|z| \text {. }
$$

Further we have

$$
-\psi_{1}(z)>0 \text { for all } z \neq 0 \text {. }
$$

Indeed, if $\psi_{1}\left(z_{0}\right)=0$ for some $z_{0} \neq 0$, then $\hat{\mu}\left(z_{0}\right) \mathrm{e}^{\mathrm{i}\left\langle w, z_{0}\right\rangle}=1$ with some $w$ and $\mu * \delta_{w}$ is concentrated on the set $\left\{x:\left\langle z_{0}, x\right\rangle=2 n \pi, n \in \mathbb{Z}\right\}$, which implies that $\psi_{1}\left(k z_{0}\right)=0$ for all $k \in \mathbb{Z}$, contrary to the strong non-lattice assumption. It follows from (3.16) and (3.17) that there is $c_{2}>0$ such that $-\psi_{1}(z)>c_{2}$ for $|z| \geqslant \varepsilon$. Since

$$
|q-\psi|^{-\eta-1}=\left(\left(q-\psi_{1}\right)^{2}+\psi_{2}^{2}\right)^{(-\eta-1) / 2} \leqslant|\psi|^{-\eta-1},
$$

we have

$$
\int_{|z| \geqslant \varepsilon} f_{b}(z)\left|(q-\psi)^{-\eta-1}\right| d z \leqslant c_{2}{ }^{-\eta-1} \int_{|z| \geqslant \varepsilon} f_{b}(z) d z,
$$

which is finite and independent of $q$. Hence

$$
\underset{q \downarrow 0}{\limsup }\left|\int_{B_{\varepsilon}} f_{b}(z)(q-\psi(z))^{-\eta-1} d z\right|<\infty .
$$

The property (3.16) implies nondegeneracy of $\mu$. Hence there are $c^{\prime}>0$ and $\varepsilon>0$ satisfying (3.7). But, recalling (3.17), we can choose $c^{\prime}>0$ such that (3.7) is true for our fixed $\varepsilon$. Using this and (3.18), we have 


$$
\begin{aligned}
\int_{|z|<\varepsilon}|z|^{2 N}\left|(q-\psi)^{-\eta-1}\right| d z & \leqslant \int_{|z|<\varepsilon}|z|^{2 N}|\psi|^{-\eta-1} d z \\
& \leqslant c^{\prime-N} \int_{|z|<\varepsilon}\left(-\psi_{1}\right)^{N}|\psi|^{-\eta-1} d z \leqslant \operatorname{const} \int_{|z|<\varepsilon}\left(-\psi_{1}\right)^{N}|\psi|^{-N-1} d z \\
& \leqslant \text { const } \int_{|z|<\varepsilon}\left(-\psi_{1}\right)|\psi|^{-2} d z,
\end{aligned}
$$

since $\eta \leqslant N$. We see that the last member is finite, noting that $\left(-\psi_{1}\right)|\psi|^{-2}=\operatorname{Re}\left(\frac{1}{-\psi}\right)$ and that $\int_{|z|<\varepsilon} \operatorname{Re}\left(\frac{1}{-\psi}\right) d z<$ $\infty$ (see [18], Corollary 37.6). It follows from this combined with (3.15) and (3.19) that

$$
\underset{q \downarrow 0}{\limsup }\left|\int_{|z|<\varepsilon}\left(\sum_{l=0}^{N-1} b^{2 l} h_{l}(z)\right)(q-\psi(z))^{-\eta-1} d z\right|<\infty .
$$

Now, suppose that there is a sequence $q_{n} \downarrow 0$ such that, when we write $I_{l, n}=\int_{B_{\varepsilon}} h_{l}(z)\left(q_{n}-\psi\right)^{-\eta-1} d z$, $\left|I_{0, n}\right| \rightarrow \infty$. Then (3.20) implies that $1+\sum_{l=1}^{N-1} b^{2 l} I_{l, n} / I_{0, n} \rightarrow 0$, which is impossible, as Lemma 3.6 says. Hence (3.12) is true for $l=0$. Next, we can show (3.12) for $l=1$ by a similar argument. Repeating this, we get (3.12) for $l=0,1, \ldots, N-1$.

Conversely, assume that (3.12) is true for $l=0,1, \ldots, N-1$. Then we get (3.20) for all $b>0$. From this (3.19) follows, and then (3.14) follows. This means (3.13) by Lemma 3.1. Hence we get (1.6).

Remark 3.7. Since $h_{l}(-z)=h_{l}(z)$ and $\psi(-z)=\overline{\psi(z)}$, the integral of $h_{l}(z) \operatorname{Im}\left((q-\psi(z))^{-\eta-1}\right)$ over $\{|z|<\varepsilon\}$ vanishes. Hence (3.12) is equivalent to

$$
\underset{q \downarrow 0}{\limsup }\left|\int_{B_{\varepsilon}} h_{l}(z) \operatorname{Re}\left((q-\psi(z))^{-\eta-1}\right) d z\right|<\infty .
$$

We can also prove that, under the same assumption as in Theorem 3.5, $\eta \in \mathfrak{T}$ if and only if

$$
\underset{q \downarrow 0}{\limsup } \int_{B_{\varepsilon}} h_{l}(z) \operatorname{Re}\left((q-\psi(z))^{-\eta-1}\right) d z<\infty
$$

for $l=0,1, \ldots, N-1$. Indeed, (3.21) clearly implies (3.22) and, conversely, if (3.22) holds for $l=0,1, \ldots, N-1$, then (3.19) holds without taking the absolute value (recalling that the integral is real), and then (3.14) is true (recalling that the integral there is real and nonnegative).

In Sato [16] it is shown that the Spitzer type criterion for recurrence and transience ([14], Theorem 16.2 or [18], Remark 37.7) does not have an analogue to judge weak and strong transience.

\section{Examples and additional results}

In the case of a symmetric Lévy process on $\mathbb{R}$, we can give criteria for $\eta \in \mathfrak{T}$ directly in terms of the Lévy measure $v$. This is an extension of Shepp's theory on random walks [20]. For a signed measure $\rho$, we denote its total variation measure by $|\rho|$. For two symmetric measures $\rho, \rho^{\prime}$ on $\mathbb{R}$, we say that $\rho$ has a bigger tail than $\rho^{\prime}$, if there is $x_{0}>0$ such that $\rho((x, \infty)) \geqslant \rho^{\prime}((x, \infty))$ for $x>x_{0}$. A symmetric measure $\rho$ is said to be quasi-unimodal if there is $x_{0}>0$ such that $\rho((x, \infty))$ is convex for $x>x_{0}$.

Theorem 4.1. Let $\left\{X_{t}\right\}$ and $\left\{Y_{t}\right\}$ be symmetric Lévy processes on $\mathbb{R}$ with Lévy measures $v_{X}$ and $v_{Y}$. When they are transient, their $\mathfrak{T}$ sets are denoted by $\mathfrak{T}_{X}$ and $\mathfrak{T}_{Y}$, respectively. 
(i) Suppose that

$$
\int_{0}^{\infty} x^{2}\left|v_{X}-v_{Y}\right|(d x)<\infty
$$

If $\left\{X_{t}\right\}$ is transient, then $\left\{Y_{t}\right\}$ is transient and $\mathfrak{T}_{X}=\mathfrak{T}_{Y}$.

(ii) Suppose that $v_{Y}$ has a bigger tail than $v_{X}$ and that $v_{Y}$ is quasi-unimodal. If $\left\{X_{t}\right\}$ is transient, then $\left\{Y_{t}\right\}$ is transient and $\mathfrak{T}_{X} \subset \mathfrak{T}_{Y}$.

Theorem 4.2. Let $\left\{X_{t}\right\}$ be a symmetric Lévy process on $\mathbb{R}$ with Lévy measure $v$.

(i) Define

$$
R(r, x)=v\left(\bigcup_{n=0}^{\infty}(2 n r+x, 2(n+1) r-x] \cap(1, \infty)\right) \text { for } r \geqslant x \geqslant 0 .
$$

Let $c>0$ be fixed. Then $\left\{X_{t}\right\}$ is transient if and only if

$$
\int_{c}^{\infty}\left(\int_{0}^{r} x R(r, x) d x\right)^{-1} d r<\infty .
$$

If $\left\{X_{t}\right\}$ is transient, then it is necessary and sufficient for $\eta \in \mathfrak{T}$ that

$$
\int_{c}^{\infty} r^{2 \eta}\left(\int_{0}^{r} x R(r, x) d x\right)^{-\eta-1} d r<\infty
$$

(ii) Assume that $v$ is quasi-unimodal. Define

$$
N(x)=v((x \vee 1, \infty)) \text { for } x \geqslant 0 .
$$

Then (4.1) is equivalent to

$$
\int_{c}^{\infty}\left(\int_{0}^{r} x N(x) d x\right)^{-1} d r<\infty
$$

(4.2) is equivalent to

$$
\int_{c}^{\infty} r^{2 \eta}\left(\int_{0}^{r} x N(x) d x\right)^{-\eta-1} d r<\infty
$$

The results on transience in the two theorems above are given in [17] and Section 38 of [18]. Based on Theorem 3.4, the assertions on the set $\mathfrak{T}$ can be proved in the same way as the proofs of similar facts on strong transience in [17]. We omit the details.

Let us give an application of Theorem 3.5.

Proposition 4.3. Let $\left\{X_{t}\right\}$ be a strongly non-lattice Lévy process on $\mathbb{R}^{d}, d \geqslant 1$. Suppose that $E\left[\left|X_{t}\right|^{2}\right]<\infty$ and $E X_{t}=0$. Then $\left\{X_{t}\right\}$ is transient with $\mathfrak{T}=\left[0, \frac{d}{2}-1\right)$ if $d \geqslant 3$; it is recurrent if $d=1$ or 2.

This is a best result in the following sense: we can find a strongly non-lattice Lévy process $\left\{X_{t}\right\}$ on $\mathbb{R}^{d}, d \geqslant 3$, satisfying $E\left[\left|X_{t}\right|^{\beta}\right]<\infty$ for $\beta \in(0,2), E X_{t}=0$, and $E\left[\left|X_{t}\right|^{2}\right]=\infty$ for $t>0$ such that $\mathfrak{T}$ is strictly larger than $\left[0, \frac{d}{2}-1\right.$ ). See Example 4.5(ii) below with $b<1-\frac{2}{d}$. 
Proof of Proposition 4.3. We use the generating triplet $(A, v, \gamma)$. Note that, for any $\beta>0, E\left[\left|X_{t}\right|^{\beta}\right]<\infty$ for $t>0$ is equivalent to $\int_{|x|>1}|x|^{\beta} \nu(d x)<\infty$ ([18], Theorem 25.3). Let $d \geqslant 3$. Let $\eta \geqslant \frac{d}{2}-1$. Since $E X_{t}=0$, we have

$$
\psi(z)=-\frac{1}{2}\langle z, A z\rangle+\int_{\mathbb{R}^{d}}\left(\mathrm{e}^{\mathrm{i}\langle z, x\rangle}-1-\mathrm{i}\langle z, x\rangle\right) v(d x) .
$$

We use $c_{1}, c_{2}, \ldots$ for positive constants. Letting $\psi_{1}$ and $\psi_{2}$ be as in the proof of Theorem 3.5, we have

$$
\begin{aligned}
& -\psi_{1}(z)=\frac{1}{2}\langle z, A z\rangle+\int_{\mathbb{R}^{d}}(1-\cos \langle z, x\rangle) v(d x) \leqslant c_{1}|z|^{2}, \\
& \left|\psi_{2}(z)\right| \leqslant \int_{\mathbb{R}^{d}}|\sin \langle z, x\rangle-\langle z, x\rangle| v(d x)=\mathrm{o}\left(|z|^{2}\right) \quad \text { as } z \rightarrow 0 .
\end{aligned}
$$

The first one is because $1-\cos \langle z, x\rangle \leqslant \frac{1}{2}|z|^{2}|x|^{2}$. The second one is because $|\sin \langle z, x\rangle-\langle z, x\rangle| \leqslant$ $\frac{1}{2}|\sin (\theta\langle z, x\rangle)||z|^{2}|x|^{2}$ with $0<\theta<1$. Hence we have, for small $\varepsilon$,

$$
\int_{|z|<\varepsilon}|\psi|^{-\eta-1} d z \geqslant c_{2} \int_{|z|<\varepsilon}|z|^{-2(\eta+1)} d z=c_{3} \int_{0}^{\varepsilon} r^{d-3-2 \eta} d r=\infty .
$$

As is shown in the proof of Theorem 3.5, the strong non-lattice property implies nondegeneracy. Thus there is $\varepsilon>0$ such that $-\psi_{1}(z) \geqslant c_{4}|z|^{2}$ for $|z|<\varepsilon$. We have

$$
\operatorname{Re}\left((q-\psi)^{-\eta-1}\right)=|q-\psi|^{-\eta-1} \cos ((-\eta-1) \arg (q-\psi))
$$

Notice that, if $\varepsilon$ is small, then

$$
|\arg (q-\psi)|=\left|\arctan \frac{-\psi_{2}}{q-\psi_{1}}\right| \leqslant\left|\frac{-\psi_{2}}{q-\psi_{1}}\right| \leqslant \frac{\left|\psi_{2}\right|}{-\psi_{1}} \leqslant \frac{\pi}{3(\eta+1)}
$$

for $|z|<\varepsilon$. Thus

$$
\int_{|z|<\varepsilon} \operatorname{Re}\left((q-\psi)^{-\eta-1}\right) d z \geqslant \frac{1}{2} \int_{|z|<\varepsilon}|q-\psi|^{-\eta-1} d z .
$$

As $q \downarrow 0$, the integral in the right-hand side increases to $\int_{|z|<\varepsilon}|\psi|^{-\eta-1} d z$, which is infinite. Hence $\eta \notin \mathfrak{T}$. This, combined with Corollary 3.3, shows that $\mathfrak{T}=\left[0, \frac{d}{2}-1\right)$. Recurrence for $d=1$ or 2 is well-known ([18], Theorems 36.7 and 37.14).

The following fact is of some interest. Its proof is a straightforward extension of that of Proposition 2.14 of [17]; we do not need the strong non-lattice property as we can use Theorem 3.2. We omit the details.

Proposition 4.4. Let $\left\{X_{t}\right\}$ and $\left\{Y_{t}\right\}$ be independent Lévy processes on $\mathbb{R}^{d}$.

(i) If $\left\{X_{t}\right\}$ is transient and symmetric, then $\left\{X_{t}+Y_{t}\right\}$ is transient and $\mathfrak{T}_{X} \subset \mathfrak{T}_{X+Y}$.

(ii) Suppose that $\left\{Y_{t}\right\}$ is identical in law with $\left\{-X_{t}\right\}$. If $\left\{X_{t}+Y_{t}\right\}$ is transient, then $\left\{X_{t}\right\}$ is transient and $\mathfrak{T}_{X+Y} \subset \mathfrak{T}_{X}$.

We give a symmetric example which can be handled by Theorem 3.4. 
Example 4.5. Let $S$ be the unit sphere $\left\{\xi \in \mathbb{R}^{d}:|\xi|=1\right\}$ and $\lambda$ a uniform measure on $S$. We consider a symmetric Lévy process on $\mathbb{R}^{d}, d \geqslant 1$, with an arbitrary Gaussian covariance matrix and with a Lévy measure $v$ satisfying

$$
\nu(B)=\int_{S} \lambda(d \xi) \int_{\mathrm{e}}^{\infty} 1_{B}(r \xi) \frac{d r}{r^{1+\alpha}(\log r)^{b}}
$$

whenever $B$ is a Borel set on $\left\{x \in \mathbb{R}^{d}:|x|>\mathrm{e}\right\}$. Here $0 \leqslant \alpha \leqslant 2 \wedge d$ and $b \in \mathbb{R}$. When $\alpha=0$, we assume $b>1$.

(i) Let $d=1$ or 2 . Assume that $\alpha=2 \wedge d$. Then, $\left\{X_{t}\right\}$ is transient with $\mathfrak{T}=\{0\}$ if $b<d-2$; it is recurrent if $b \geqslant d-2$.

(ii) Let $d \geqslant 3$. Assume that $\alpha=2$. Then $\left\{X_{t}\right\}$ is transient with $\mathfrak{T}=\left[0, \frac{d}{2}-1\right)$ if $b \geqslant 1-\frac{2}{d}$; it is transient with $\mathfrak{T}=\left[0, \frac{d}{2}-1\right]$ if $b<1-\frac{2}{d}$.

(iii) Let $d \geqslant 1$. Let $0<\alpha<d \wedge 2$. Then $\left\{X_{t}\right\}$ is transient with $\mathfrak{T}=\left[0, \frac{d}{\alpha}-1\right)$ if $b \geqslant-\frac{\alpha}{d}$; it is transient with $\mathfrak{T}=\left[0, \frac{d}{\alpha}-1\right]$ if $b<-\frac{\alpha}{d}$.

(iv) Let $d \geqslant 1$. Assume that $\alpha=0$ and $b>1$. Then $\left\{X_{t}\right\}$ is transient with $\mathfrak{T}=[0, \infty)$.

For a proof, note that

$$
\begin{aligned}
-\psi(z) & =\int_{S} \lambda(d \xi) \int_{\mathrm{e}}^{\infty}\left(1-\cos \left(r|z|\left|\xi_{1}\right|\right)\right)(\log r)^{-b} r^{-1-\alpha} d r+\mathrm{O}\left(|z|^{2}\right) \\
& =|z|^{\alpha} \int_{S} \lambda(d \xi)\left|\xi_{1}\right|^{\alpha} \int_{\mathrm{e}|z|\left|\xi_{1}\right|}^{\infty}(1-\cos u)\left(\log \frac{u}{|z|\left|\xi_{1}\right|}\right)^{-b} u^{-1-\alpha} d u+\mathrm{O}\left(|z|^{2}\right),
\end{aligned}
$$

where $\xi=\left(\xi_{j}\right)_{1 \leqslant j \leqslant d} \in S$. The details are omitted.

\section{One-dimensional Lévy processes with positive mean}

We give some results on the set $\mathfrak{T}$ and the set $\left\{\eta: E\left[L_{(-\infty, a]}^{\eta}\right]<\infty\right\}$ for one-dimensional Lévy processes with positive mean.

Theorem 5.1. Let $\left\{X_{t}\right\}$ be a Lévy process on $\mathbb{R}$ such that $E\left[\left|X_{1}\right|\right]<\infty$ and $E X_{1}>0$. Let $\eta>0$ and $a \geqslant 0$. Then the following three conditions are equivalent:

$$
\begin{aligned}
& E\left[\left|X_{1} \wedge 0\right|^{1+\eta}\right]<\infty, \\
& \eta \in \mathfrak{T}, \\
& \left.E\left[L_{(-\infty, a]}\right]^{\eta}\right]<\infty
\end{aligned}
$$

Note that $\lim _{t \rightarrow \infty} X_{t}=\infty$ a.s. since $E X_{1}>0$. If we assume $E X_{1}<0$ and $a \leqslant 0$ instead of $E X_{1}>0$ and $a \geqslant 0$, then we have the dual statement with (5.1) and (5.3) replaced by $E\left[\left(X_{1} \vee 0\right)^{1+\eta}\right]<\infty$ and $\left.E\left[L_{[a, \infty}\right)^{\eta}\right]<\infty$, respectively. The condition (5.1) can be written in terms of the Lévy measure $v$ of $\left\{X_{t}\right\}$. In fact, by [18] Theorem 25.3 and Proposition 25.4, (5.1) holds if and only if

$$
\int_{(-\infty,-1)}|x|^{1+\eta} v(d x)<\infty .
$$

If we assume that $E\left[\left|X_{1}\right|^{1+\delta}\right]<\infty$ for some $\delta>0$ and that $\left\{X_{t}\right\}$ is strongly non-lattice, we can prove the equivalence of (5.1) and (5.2) in Theorem 5.1 by applying Theorem 3.5 with careful use of residue calclus. In this 
way we arrived at the condition (5.1) expressed in the form of (5.4). But we had not been able to prove Theorem 5.1 in its generality until we came to know Janson's work [8] on random walks.

Proof of Theorem 5.1. Let $\left\{S_{n}: n=0,1, \ldots\right\}$ be the discrete skeleton of $\left\{X_{t}\right\}$. That is, $\left\{S_{n}\right\}$ is the random walk defined by $S_{n}=X_{n}$. Let $L_{B}^{0}=\sup \left\{n \geqslant 0: S_{n} \in B\right\}$. Theorem 1 of Janson [8] tells us that each of the following two conditions is equivalent to $(5.1)$ :

$$
\begin{aligned}
& E\left[\left(L_{(-\infty, a]}^{0}\right)^{\eta}\right]<\infty, \\
& \sum_{n=1}^{\infty} n^{\eta-1} P\left(S_{n} \leqslant a\right)<\infty .
\end{aligned}
$$

Thus, in order to show Theorem 5.1, it is enough to prove the equivalence of (5.2), (5.3), and (5.5).

First, assume (5.2) and let us prove (5.5). Let $\mu^{t}$ and $U$ be as in Section 2. Let $\mathfrak{G}$ be the smallest additive subgroup of $\mathbb{R}$ that contains the support $\Sigma$. If $\mathfrak{G}=\mathbb{R}$, then let $B=(-1 / 2,1 / 2]$. If $\mathfrak{G}=c \mathbb{Z}$ with some $c>0$, then let $B=(-c / 2, c / 2]$. Using Lemma 2.3, we get

$$
\begin{aligned}
\infty & >\int_{0}^{\infty} t^{\eta} \mu^{t}(B) d t=\eta \int_{0}^{\infty} t^{\eta-1} d t \int_{t}^{\infty} \mu^{s}(B) d s \\
& =\eta \int_{0}^{\infty} t^{\eta-1} d t \int_{0}^{\infty} \mu^{t+s}(B) d s \\
& =\eta \int_{0}^{\infty} t^{\eta-1} d t \int_{0}^{\infty} d s \int_{-\infty}^{\infty} \mu^{s}(B-y) \mu^{t}(d y) \\
& =\eta \int_{0}^{\infty} t^{\eta-1} d t \int_{-\infty}^{\infty} U(B-y) \mu^{t}(d y) .
\end{aligned}
$$

If $\mathfrak{G}=\mathbb{R}$, then the renewal theorem ([1], p. 38 or [18], p. 271) tells us that $U(B-y) \rightarrow 1 / E\left[X_{1}\right]$ as $y \rightarrow-\infty$. Similarly, if $\mathfrak{G}=c \mathbb{Z}$, then $U(B-y) \rightarrow c / E\left[X_{1}\right]$ as $y \rightarrow-\infty$. In any case, there are $c_{1}>0$ and $N>0$ such that $U(B-y) \geqslant c_{1}$ for all $y \leqslant-N$. It follows that

$$
\int_{0}^{\infty} t^{\eta-1} \mu^{t}(-\infty,-N] d t<\infty
$$

On the other hand, (5.2) implies that $\int_{0}^{\infty} t^{\eta} \mu^{t}(-N, b] d t<\infty$ for any $b \geqslant 0$ by Lemma 2.3. Hence,

$$
\int_{0}^{\infty} t^{\eta-1} \mu^{t}(-\infty, b] d t<\infty \quad \text { for any } b \geqslant 0 .
$$

Choose $b^{\prime}>0$ such that $P\left(\sup _{0 \leqslant s \leqslant 1}\left|X_{s}\right| \leqslant b^{\prime}\right)>0$. Denote this probability by $c_{2}$. If $n \leqslant t \leqslant n+1$, then

$$
\begin{aligned}
\mu^{t}\left(-\infty, b+b^{\prime}\right] & =P\left(X_{n}+X_{t}-X_{n} \leqslant b+b^{\prime}\right) \\
& \geqslant P\left(X_{n} \leqslant b\right) P\left(\sup _{n \leqslant s \leqslant n+1}\left|X_{s}-X_{n}\right| \leqslant b^{\prime}\right) \\
& =c_{2} P\left(X_{n} \leqslant b\right) .
\end{aligned}
$$


Thus it follows from (5.7) that

$$
\sum_{n=1}^{\infty} n^{\eta-1} P\left(X_{n} \leqslant b\right)<\infty \quad \text { for all } b \geqslant 0 .
$$

In particular (5.6) is true. Hence we get (5.5) by Janson's result mentioined above.

Next, assume (5.5) and let us prove (5.3). We use $b^{\prime}$ and $c_{2}$ in the discussion above. Since Janson's result says that the condition (5.5) does not depend on $a$, we have

$$
E\left[\left(L_{\left(-\infty, a^{\prime}\right]}^{0}\right)^{\eta}\right]<\infty \text { for all } a^{\prime} \geqslant 0 .
$$

Notice that

$$
P\left(L_{(-\infty, a]}>t\right)=P\left(X_{s} \leqslant a \text { for some } s>t\right)=E\left[P^{X_{t}}\left(T_{(-\infty, a]}<\infty\right)\right],
$$

where, as before, the superscript $y$ in $P^{y}$ denotes the starting point. For any $y \in \mathbb{R}$ we have, for $T=T_{(-\infty, a]}$,

$$
\begin{aligned}
& P^{y}\left(T<\infty \text { and } X_{T+s} \leqslant a+b^{\prime} \text { for all } s \in[0,1]\right) \\
& \quad=E^{y}\left[P^{X_{T}}\left(X_{s} \leqslant a+b^{\prime} \text { for all } s \in[0,1]\right) ; T<\infty\right] \\
& \geqslant P^{a}\left(X_{s} \leqslant a+b^{\prime} \text { for all } s \in[0,1]\right) P^{y}(T<\infty) \\
& \quad \geqslant c_{2} P^{y}(T<\infty) .
\end{aligned}
$$

Hence

$$
\begin{aligned}
P\left(L_{(-\infty, a]}>t\right) & \leqslant c_{2}^{-1} E\left[P^{X_{t}}\left(T<\infty \text { and } X_{T+s} \leqslant a+b^{\prime} \text { for all } s \in[0,1]\right)\right] \\
& \leqslant c_{2}^{-1} P\left(X_{n} \leqslant a+b^{\prime} \text { for some integer } n>t\right) \\
& =c_{2}^{-1} P\left(L_{\left(-\infty, a+b^{\prime}\right]}^{0}>t\right) .
\end{aligned}
$$

It follows that

$$
\begin{aligned}
E\left(L_{(-\infty, a]}^{\eta}\right) & =\eta \int_{0}^{\infty} P\left(L_{(-\infty, a]}>t\right) t^{\eta-1} d t \\
& \leqslant \eta c_{2}^{-1} \int_{0}^{\infty} P\left(L_{\left(-\infty, a+b^{\prime}\right]}^{0}>t\right) t^{\eta-1} d t \\
& =c_{2}^{-1} E\left[\left(L_{\left(-\infty, a+b^{\prime}\right]}^{0}\right)^{\eta}\right],
\end{aligned}
$$

which is finite by (5.8). Thus we get (5.3).

Assume (5.3). Then (5.5) holds, since $L_{(-\infty, a]}^{0} \leqslant L_{(-\infty, a]}$. Hence (5.8) holds. The proof that (5.5) implies (5.3) now shows that $\left.E\left[L_{\left(-\infty, a^{\prime}\right]}\right]^{\eta}\right]<\infty$ for all $a^{\prime} \geqslant 0$. If $a^{\prime}>0$, then $L_{\left(-a^{\prime}, a^{\prime}\right)} \leqslant L_{\left(-\infty, a^{\prime}\right]}$. Thus we get (5.2). This completes the proof of the equivalence of (5.2), (5.3), and (5.5).

Using Remark 3.1 of [8], we can check that Theorem 5.1 remains true if we replace $a \geqslant 0$ by $a \in \mathbb{R}$. Here we define $L_{B}=0$ whenever $X_{t} \notin B$ for all $t \geqslant 0$.

As a direct consequence of Theorem 5.1 we get the following, which extends Proposition 3.2 of [16] to the best possible.

Corollary 5.2. (i) If $\left\{X_{t}\right\}$ is a transient Lévy process on $\mathbb{R}$ satisfying $E\left[\left|X_{1}\right|^{2}\right]<\infty$, then it is strongly transient. (ii) There is a weakly transient Lévy process $\left\{X_{t}\right\}$ on $\mathbb{R}$ such that, for every $c \in(0,2), E\left[\left|X_{1}\right|^{c}\right]<\infty$. 
Here is an application in the multi-dimensional case.

Corollary 5.3. Let $\left\{X_{t}\right\}$ be a transient Lévy process on $\mathbb{R}^{d}, d \geqslant 2$, such that $E\left[\left|X_{1}\right|\right]<\infty$. Suppose that $E X_{1}=\tau \neq 0$. Let $\eta>0$. If $E\left[\left|\left\langle\tau, X_{1}\right\rangle \wedge 0\right|^{1+\eta}\right]<\infty$, then $\eta \in \mathfrak{T}$.

To see this, apply Theorem 5.1 to the process $Y_{t}=\left\langle\tau, X_{t}\right\rangle$.

The equivalence of (5.1), (5.2), and (5.3) fails to hold for some process with $E X_{1}=\infty$, as the example below shows.

Example 5.4. Assume that $X_{t}=Y_{t}+Z_{t}$, where $\left\{Y_{t}\right\}$ and $\left\{Z_{t}\right\}$ are independent, $\left\{Y_{t}\right\}$ is a strictly $\alpha$-stable suborinator, $0<\alpha<1$, and $\left\{Z_{t}\right\}$ is a strictly $\beta$-stable process, $1<\beta<2$, with Lévy measure concentrated on the negative axis. Then $E X_{1}=\infty$ and thus $\lim _{t \rightarrow \infty} X_{t}=\infty$ a.s. For $\eta>0$ and $a \geqslant 0$, we can show the following:

$$
\begin{array}{rll}
\eta \in \mathfrak{T} & \text { if and only if } & \eta<(1+\beta) / \alpha-2 ; \\
E\left[L_{\left.(-\infty, a]^{\eta}\right]<\infty}\right. & \text { if and only if } & \eta<\beta / \alpha-1 ; \\
E\left[\left|X_{1} \wedge 0\right|^{1+\eta}\right]<\infty & \text { if and only if } & \eta<\beta-1 .
\end{array}
$$

Proof is as follows. The Lévy measures $v_{Y}$ and $v_{Z}$ of $\left\{Y_{t}\right\}$ and $\left\{Z_{t}\right\}$ are concentrated on $(0, \infty)$ and $(-\infty, 0)$, respectively, and have the form $v_{Y}(d x)=c_{1} x^{-1-\alpha} d x$ and $v_{Z}(d x)=c_{2}|x|^{-1-\beta} d x$ with positive constants $c_{1}$ and $c_{2}$. Hence (5.11) follows from (5.4). To show (5.9), we use Lemma 2.3. Let $p_{t}^{X}(x), p_{t}^{Y}(x), p_{t}^{Z}(x)$, be the densities of the distributions of $X_{t}, Y_{t}, Z_{t}$ for $t>0$. These are smooth bounded functions on $\mathbb{R}$. Strict stability implies that

$$
p_{t}^{Y}(x)=t^{-1 / \alpha} p^{Y}\left(t^{-1 / \alpha} x\right), \quad p_{t}^{Z}(x)=t^{-1 / \beta} p^{Z}\left(t^{-1 / \beta} x\right),
$$

where $p^{Y}(x)=p_{1}^{Y}(x)$ and $p^{Z}(x)=p_{1}^{Z}(x)$. Thus

$$
\begin{aligned}
p_{t}^{X}(x) & =t^{-1 / \alpha-1 / \beta} \int_{-\infty}^{x} p^{Y}\left(t^{-1 / \alpha}(x-y)\right) p^{Z}\left(t^{-1 / \beta} y\right) d y \\
& =t^{-1 / \alpha} \int_{-\infty}^{t^{-1 / \beta} x} p^{Y}\left(t^{-1 / \alpha} x-t^{-1 / \alpha+1 / \beta} y\right) p^{Z}(y) d y
\end{aligned}
$$

It is known that, for any $\gamma>0, x^{-\gamma} p^{Y}(x) \rightarrow 0$ as $x \downarrow 0$ and that there are positive constants $c_{3}, c_{4}$ such that

$$
p^{Y}(x) \sim c_{3}(1+x)^{-1-\alpha} \quad \text { as } x \rightarrow \infty, \quad p^{Z}(x) \sim c_{4}(1+|x|)^{-1-\beta} \quad \text { as } x \rightarrow-\infty
$$

(see [18], p. 88). Let

$$
q_{t}(x)=t^{-1 / \alpha} \int_{-t^{1 / \alpha-1 / \beta}}^{t^{-1 / \beta} x} p^{Y}\left(t^{-1 / \alpha} x-t^{-1 / \alpha+1 / \beta} y\right) p^{Z}(y) d y .
$$

Then, choosing $\gamma>\beta$, we can show that, for any $a>0$, there is $c_{5}$ such that

$$
\sup _{|x|<a} q_{t}(x) \leqslant c_{5} t^{1-(1+\beta) / \alpha} \quad \text { for all large } t .
$$

Furthermore, there are positive constants $c_{6}, c_{7}$ such that

$$
c_{6} t^{1-(1+\beta) / \alpha} \leqslant p_{t}^{X}(x)-q_{t}(x) \leqslant c_{7} t^{1-(1+\beta) / \alpha} \quad \text { for all large } t
$$

uniformly in $|x|<a$. Hence, checking (1.6) or (1.7), we get (5.9). 
Proof of (5.10) is as follows. Let $\left\{S_{n}\right\}$ be the discrete skeleton of $\left\{X_{t}\right\}$ as in the proof of Theorem 5.1. We use a result of Kesten and Maller [9]. They extended Janson's work [8] and studied random walks with infinite mean, exploiting Erickson's method [4]. Let $\eta>0$ and $a \geqslant 0$. Define

$$
A(x)=\int_{0}^{x} P\left(X_{1}>y\right) d y-\int_{0}^{x} P\left(X_{1} \leqslant-y\right) d y \quad \text { for } x>0 .
$$

Since $S_{n} \rightarrow \infty$ a.s., Theorem 2.1 of [9] tells us that $E\left[\left(L_{(-\infty, a]}^{0}\right)^{\eta}\right]<\infty$ if and only if there is $x_{0}>0$ such that

$$
A(x)>0 \text { for } x \geqslant x_{0} \text { and } \int_{\left[x_{0}, \infty\right)}\left(\frac{x}{A(x)}\right)^{1+\eta} P\left(-X_{1} \in d x\right)<\infty .
$$

By a result of Sgibnev [19], there are positive constants $c_{8}, c_{9}$ such that

$$
P\left(X_{1}>x\right) \sim c_{8} x^{-\alpha} \text { as } x \rightarrow \infty, \quad P\left(X_{1}<x\right) \sim c_{9}|x|^{-\beta} \quad \text { as } x \rightarrow-\infty .
$$

It follows that $A(x) \sim c_{10} x^{1-\alpha}$ as $x \rightarrow \infty$ with some $c_{10}>0$. Thus the integral condition in (5.12) is written as

$$
\int_{x_{0}}^{\infty} x^{(1+\eta) \alpha} P\left(-X_{1} \in d x\right)<\infty
$$

or, equivalently,

$$
\int_{x_{0}}^{\infty} P\left(-X_{1}>x\right) x^{(1+\eta) \alpha-1} d x<\infty
$$

that is, $\eta<\beta / \alpha-1$. Thus we see that $E\left[\left(L_{(-\infty, a]}^{0}\right)^{\eta}\right]<\infty$ if and only if $\eta<\beta / \alpha-1$. The condition that $E\left[\left(L_{(-\infty, a]}^{0}\right)^{\eta}\right]<\infty$ does not depend on $a$. Arguing as in the proof of Theorem 5.1, we can show that $E\left[\left(L_{(-\infty, a]}^{0}\right)^{\eta}\right]<\infty$ if and only if $E\left[L_{(-\infty, a]}^{\eta}\right]<\infty$.

\section{Acknowledgement}

The authors thank a referee for pointing out the relevance of the paper to the works of Janson [8] and Kesten and Maller [9] on random walks. Section 5 was rewritten after the comment.

\section{References}

[1] J. Bertoin, Lévy Processes, Cambridge Univ. Press, Cambridge, 1996.

[2] R.M. Blumenthal, R.K. Getoor, Markov Processes and Potential Theory, Academic Press, New York, 1968.

[3] D.A. Dawson, L.G. Gorostiza, A. Wakolbinger, Occupation time fluctuations in branching systems, J. Theoret. Probab. 14 (2001) $729-796$.

[4] K.B. Erickson, The strong law of large numbers when the mean is undefined, Trans. Amer. Math. Soc. 185 (1973) $371-381$.

[5] R.K. Getoor, Some asymptotic formulas involving capacity, Z. Wahrsch. Verw. Gebiete 4 (1965) 248-252.

[6] J. Hawkes, Moments of last exit times, Mathematika 24 (1977) 266-269.

[7] N. Jain, W.E. Pruitt, The range of random walk, in: Proc. Sixth. Berkeley Symp. Math. Statist. Probab., vol. 3, Univ. Calif. Press, Berkeley, 1972, pp. 31-50.

[8] S. Janson, Moments for first-passage and last-exit times, the minimum, and related quantities for random walks with positive drift, Adv. Appl. Probab. 18 (1986) 865-879. 
[9] H. Kesten, R.A. Maller, Two renewal theorems for general random walks tending to infinity, Probab. Theory Related Fields 106 (1996) $1-38$.

[10] K. Petersen, Ergodic Theory, Cambridge Univ. Press, Cambridge, 1983.

[11] S.C. Port, Limit theorems involving capacities, J. Math. Mech. 15 (1966) 805-832.

[12] S.C. Port, Limit theorems for transient Markov chains, J. Combinatorial Theory 2 (1967) 107-128.

[13] S.C. Port, Hitting times for transient stable processes, Pacific J. Math. 21 (1967) 161-165.

[14] S.C. Port, C.J. Stone, Infinitely divisible processes and their potential theory, I and II, Ann. Inst. Fourier (Grenoble) 21 (2) (1971) 157-275 and (4) 179-265.

[15] R.T. Rockafellar, Convex Analysis, Princeton Univ. Press, Princeton, NJ, 1970.

[16] K. Sato, Criteria of weak and strong transience for Lévy processes, in: S. Watanabe, et al. (Eds.), Probability Theory and Mathematical Statistics, Proc. Seventh Japan-Russia Symp., World Scientific, Singapore, 1996, pp. 438-449.

[17] K. Sato, Time evolution of Lévy processes, in: N. Kono, N.R. Shieh (Eds.), Trends in Probability and Related Analysis, Proc. SAP '96, World Scientific, Singapore, 1997, pp. 35-82.

[18] K. Sato, Lévy Processes and Infinitely Divisible Distributions, Cambridge Univ. Press, Cambridge, 1999.

[19] M.S. Sgibnev, Asymptotics of infinitely divisible distributions on R, Siberian Math. J. 31 (1990) $115-119$.

[20] L.A. Shepp, Symmetric random walk, Trans. Amer. Math. Soc. 104 (1962) 144-153.

[21] F. Spitzer, Electrostatic capacity, heat flow, and Brownian motion, Z. Wahrsch. Verw. Gebiete 3 (1964) $110-121$.

[22] J. Takeuchi, Moments of the last exit times, Proc. Japan Acad. 43 (1967) 355-360.

[23] J. Takeuchi, T. Yamada, S. Watanabe, Antei katei (Stable processes), in: Seminar on Probability, vol. 13, Kakuritsuron Seminar, Japan, 1962 (in Japanese).

[24] K. Yamamuro, On transient Markov processes of Ornstein-Uhlenbeck type, Nagoya Math. J. 149 (1998) 19-32. 